Article

\title{
Effect of Gold Nanoparticle Conjugation on Peptide Dynamics and Structure
}

\section{Kuo Hao Lee and F. Marty Ytreberg *}

Department of Physics, University of Idaho, Moscow, ID 83844, USA;

E-Mail: khlee@vandals.uidaho.edu

* Author to whom correspondence should be addressed; E-Mail: ytreberg@uidaho.edu; Tel.: +1-208-885-6908; Fax: +1-208-885-4055.

Received: 31 January 2012; in revised form: 10 March 2012 / Accepted: 19 March 2012 /

Published: 23 March 2012

\begin{abstract}
Molecular dynamics simulations were used to characterize the structure and dynamics for several peptides and the effect of conjugating them to a gold nanoparticle. Peptide structure and dynamics were compared for two cases: unbound peptides in water, and peptides bound to the gold nanoparticle surface in water. The results show that conjugating the peptides to the gold nanoparticle usually decreases conformational entropy, but sometimes increases entropy. Conjugating the peptides can also result in more extended structures or more compact structures depending on the amino acid sequence of the peptide. The results also suggest that if one wishes to use peptide-nanoparticle conjugates for drug delivery it is important that the peptides contain secondary structure in solution because in our simulations the peptides with little to no secondary structure adsorbed to the nanoparticle surface.
\end{abstract}

Keywords: nanoparticle-peptide interactions; molecular dynamics; drug delivery; nano-conjugate

PACS Codes: 81.07.Nb, 87.10.Tf 


\section{Introduction}

Nanoparticles (NPs) are of interest due to their use in applications such as sensing [1], imaging [2-4], drug delivery, novel therapy [5], and control of protein structure and activity [6]. In the emerging field of nanomaterial-biomolecule research, gold NPs are ideal drug-delivery agents because of their well-known chemical inertness and their minimal toxicity [7,8]. Functionalized gold NPs often conjugate with certain proteins, antibodies, or peptides $[9,10]$. Thus, these conjugates can be designed to target specific cells such as cancer cells and then deliver drugs to the targeted cells, which can reduce the dose and thus possible side effects. Many technologies based on biomolecule conjugation to NP have been developed in the past two decades. For example, aptamer bioconjugated NPs have shown a very high specificity for drug delivery in prostate cancer chemotherapy [11,12]. Platinum-based anticancer drugs on gold NPs have demonstrated an unusual ability to penetrate the nucleus in lung cancer cells [13].

Several studies have shown that it is more effective to use gold NPs as drug delivery agents than using the drugs via traditional means. Cheng et al. found that use of a drug-gold NP conjugate reduced drug delivery times significantly compared to the free drug [14]. Thomas and Klibanov showed that conjugating polyethylenimine chains to gold NPs enhanced the ability of polyethylenimine to transfer plasmid DNA into mammalian cells [15]. Joshi et al. observed a significant reduction of blood glucose levels when insulin was delivered using gold NPs as carriers by the transmucosal route in diabetic rats [16].

In order to more effectively utilize NPs for drug delivery it is important to understand and visualize how biomolecules interact with NPs. It is known that conjugation of proteins to NPs can affect the protein structure and function. Aubin-Tam and Hamad-Schifferli have shown that the structure and function are influenced by the chemistry of the NP ligand, the NP size, the NP material, the stoichiometry of the conjugates, and the labeling site on the protein and the nature of the linkage $[17,18]$. They also showed that surface-coating ligands on the NPs are labile and can adopt multiple conformations. Verma et al. have observed that while initial electrostatic complementarity mediates binding, further stabilization is achieved through additional favorable interactions on the surface of NP and the peptide [19]. It is also known that the ligands can rearrange to optimize the interaction with a protein [20].

Computer simulation has emerged as a particularly valuable tool for characterizing and visualizing biomolecule-NP conjugates due to the difficulty in obtaining actual experimental data for such systems $[21,22]$. Schulten and collaborators used molecular dynamics to predict the structure of an engineered polypeptide on a gold surface [23]. Simulations by Hoefling et al. showed that the binding affinities are dependent on the chemical character of the amino acids when adsorbed to a gold surface [24]. Verde et al. performed simulations to investigate the adsorption and mobility of peptides on a gold surface [25]. Duchesne et al. devised a method to estimate the proximity of peptides on a gold NP surface. Other researchers have used simulation to investigate DNA conformations while bound to a gold surface [26-28].

For the current study we hypothesized that conjugating peptides to gold NPs induces changes in both the peptide structure and peptide dynamics. We studied six peptides: two sequences that were used in a cellular uptake study by Hill and colleagues [29], and the other four sequences which are 
currently under investigation by Hill and colleagues [30]. We performed molecular dynamics simulations for the six peptides under two conditions: (i) unbound peptides in water with no gold NP present; and (ii) peptides bound to a gold NP in water. We then compared the structure and dynamics of the peptides under these conditions. Results show that conjugating the peptides to the gold nanoparticle typically decreases conformational flexibility, but that increases in flexibility are also possible. The difference in flexibility upon conjugation depends on the amino acid sequence of the peptide. We also find that conjugation can result in more extended structures or more compact structures depending on the amino acid sequence of the peptide. Finally, the simulations reveal that peptides with no secondary structure in solution tend to adsorb to the nanoparticle surface at multiple points along the peptide, potentially inhibiting their ability to have specific interactions with cellular media. This suggests that if one wishes to use peptide-nanoparticle conjugates for drug delivery it is important that the peptides contain secondary structure in solution.

\section{Materials and Methods}

We used six different peptides for this study: kdel cgy peptide (KC), kdel cgy labeled by lissamine (KCL), muscle binding peptide (MB), muscle binding peptide labeled by lissamine (MBL), negative control peptide (NC), and negative control peptide labeled by lissamine (NCL) [29]. The corresponding peptide amino acid sequences are:

\section{KC: H2N-CGYRQSDIDTHNRIKDEL-OH \\ KCL: H2N-CGY[KLiss]RQSDIDTHNRIKDEL-OH \\ MB: H2N-CGYQNASSLNIA-OH \\ MBL: H2N-CGY[KLiss]QNASSLNIA-OH \\ NC: H2N-CGYHGEGHGEGHGEGK-OH \\ NCL: H2N-CGY[KLiss]HGEGHGEGHGEGK-OH}

For each peptide we used PEP-FOLD to generate predicted structures [31], we then constructed two simulation systems for each peptide: a single unbound peptide in water and a single peptide bound to a gold NP in water (see Figure 1).

Figure 1. Simulation test cases used in this study. (a) Unbound: single peptide in water. (b) Conjugated: peptide conjugated to the gold NP in water. For the conjugated case the sulfur atom (yellow sphere) is covalently bonded to the NP.

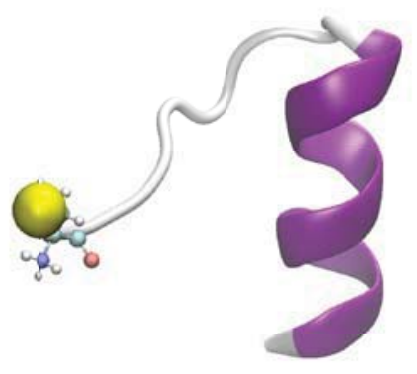

(a) Unbound

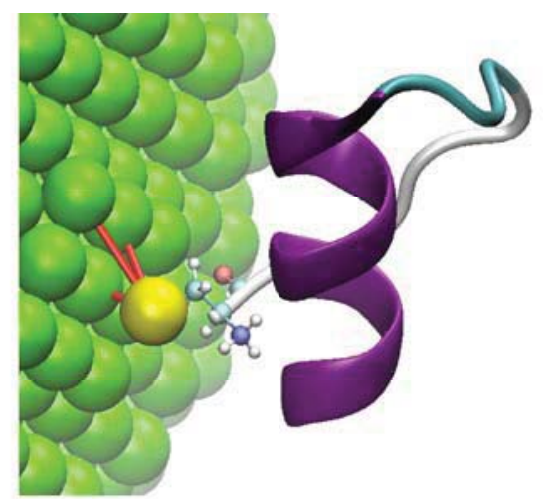

(b) Conjugated 
For the unbound case the peptides were solvated in a $50.0 \AA$ length cubic water box. Each system was then ionized with $0.5 \mathrm{~mol} / \mathrm{L}$ ion concentration using a mixture of $\mathrm{Na}^{+}$and $\mathrm{Cl}^{-}$ions such that each system had zero net charge.

For the case of the peptide bound to the gold NP the peptide was first placed in water such that the sulfur atom in the cysteine was $3.0 \AA$ away from the surface of a $5.0 \mathrm{~nm}$ diameter gold NP. The coordinates for the gold atoms were obtained by trimming a (111) symmetry crystal structure to form a $5.0 \mathrm{~nm}$ diameter sphere. The distance of $3.0 \AA$ was chosen so that the system energy could be minimized effectively since Jiang et al. showed that the S-Au covalent bond length is around $2.8 \AA$ (Figure 1b) [32]. To reduce computational cost most of the gold atoms that could not interact with the peptide atoms were not included in the simulation. The system was then solvated in a rectangular water box and ionized with $0.5 \mathrm{~mol} / \mathrm{L}$ ion concentration using a mixture of $\mathrm{Na}^{+}$and $\mathrm{Cl}^{-}$ions such that each system had zero net charge. The box size was chosen such that the distance between the edge of the box and the nearest gold or peptide atoms was at least $24.0 \AA$ (twice the interaction cutoff distance).

All the molecular dynamics simulations were performed using NAMD 2.7b1 [33] with the TIP3P water model [34] and CHARMM force field [35,36]. There are several forcefields for peptide-gold interactions that have been reported in the literature (e.g., [37,38]). We used parameters describing the interactions for Au-Au and S-Au from Vila Verde et al. and Miao and Seminario [25,36]. Assigning these forcefield parameters neglects possible confinement effects on NP electronic structure that could change NP reactivity. These parameters also ignore possible interactions between the peptide and NP features such as edges and vertexes. For our simulations partial charges for the gold atoms were set to zero and the atoms were forced to remain in fixed positions during the simulation. For each of the two cases (unbound and conjugated) minimization was performed for 1,000 steps, followed by $20.0 \mathrm{~ns}$ of equilibrium, and $20.0 \mathrm{~ns}$ of production simulation. For equilibration and production Langevin dynamics [39] was used with a constant temperature of $300 \mathrm{~K}$ and the pressure was maintained at $1.0 \mathrm{~atm}[40,41]$. The SHAKE algorithm was implemented to allow a 2.0 fs timestep [42]. Particle mesh Ewald was utilized for electrostatics with a real-space cutoff of $12 \AA$ [43]. Van der Waals interactions were cut off at $12 \AA$ with a switching function between 10 and $12 \AA$.

In order to compare structures and dynamics between the unbound and conjugated cases for each peptide we used the trajectory produced during the $20 \mathrm{~ns}$ production simulation to analyze the solvent accessible surface area (SASA), root-mean-square fluctuation (RMSF) and conformational entropy, and performed clustering for the production simulations. The SASA and RMSF were measured by analyzing the molecular dynamics trajectories obtained from the production simulations using VMD [44]. SASA is the solvent accessible surface area of the peptide and the RMSF of the C $\alpha$ quantifies the peptide dynamics. Before performing the RMSF analysis all the trajectory frames were aligned to the last frame of the trajectory. Clustering of the trajectory structures was performed by first generating the RMSD matrix, and then using the cutree function in the software package R [45]. The number of clusters used for our analysis was chosen such that any increase in the number of clusters did not change the largest cluster. For each peptide, the structure in the largest cluster with a SASA value closest to the average SASA was then chosen to represent the most commonly seen type of structure in the simulations. To estimate the uncertainty of SASA and conformational entropy, the production simulation trajectory was divided into 20 equal size pieces, and the standard deviation of those pieces was calculated and used as an estimate of the uncertainty for each measure. The 
conformational entropy associated with the backbone atoms was estimated via a quasiharmonic implemented in the $g$ anaeig program in GROMACS [46]. In this approximation, each atom $i$ is considered as a quantum-mechanical harmonic oscillator of frequency $\omega_{i}$ [47-49]. For this study the entropy was estimated after aligning each trajectory frame to the last frame of the trajectory to remove possible entropic contributions from the overall translational and rotational motion of the peptides.

\section{Results and Discussion}

To study the effects of peptide-nanoparticle conjugation we used molecular dynamics simulation to compare the dynamics and structure of six different peptides under two sets of conditions shown in Figure 1: (i) a single unbound peptide in water with no gold NP present; and (ii) a single peptide bound to a gold NP in water. The motivation for this study was the hypothesis that both the peptide structure and dynamics will change due to the peptide-NP interactions. While $40 \mathrm{~ns}$ of simulation is not long enough to fully explore all of the conformational space available to the peptides, it does appear to be sufficient to obtain robust results for the properties reported here based on the reasonable uncertainties obtained from block averaging.

Figure 2 illustrates the peptide starting structures used for this study, and also the most common structure for unbound and conjugated peptides during the production simulations (representative structure from largest cluster). The figure shows that, consistent with our hypothesis, conjugating the peptides to the gold NP does change the peptide structure. These results also suggest that the peptides tend to lose some secondary structure when conjugated to the NP.

Figure 2. Initial simulation structures used in this study and the most common peptide structures in our molecular dynamics. Initial structures were generated using the PEP-FOLD server [31]. The most common simulation structures are shown for both unbound and conjugated cases. The spheres (yellow) indicate the location of the sulfur atoms that are bonded to the gold surface and are part of the cysteine residues (blue).

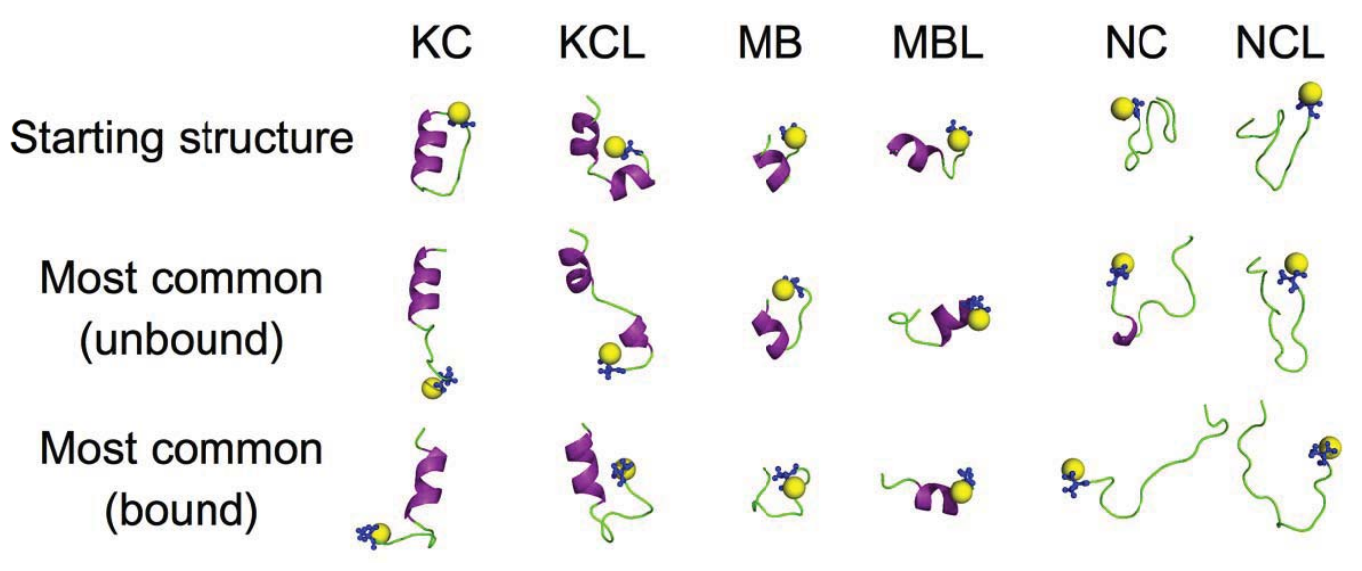

Figure 3 shows the SASA for unbound and conjugated peptides. The SASA measurement provides a measure of the compactness of the peptides [50]. The NC and NCL peptides have significantly increased SASA when conjugated with gold NP. By contrast, the other peptides have similar or slightly smaller SASA when conjugated. Thus, the results suggest that the peptides with little to no 
secondary structure become more extended when bound to the gold NP, and that the peptides with secondary structure do not significantly change compactness due to conjugation.

Figure 3. Average solvent accessible surface area (SASA) for unbound (white) and conjugated (grey) cases for each peptide. The SASA is a measure of how extended the peptide conformation is during the simulations. Averages (bars) were computed for the $20 \mathrm{~ns}$ production simulation and uncertainties (error bars) were estimated as the standard deviation of block averages.

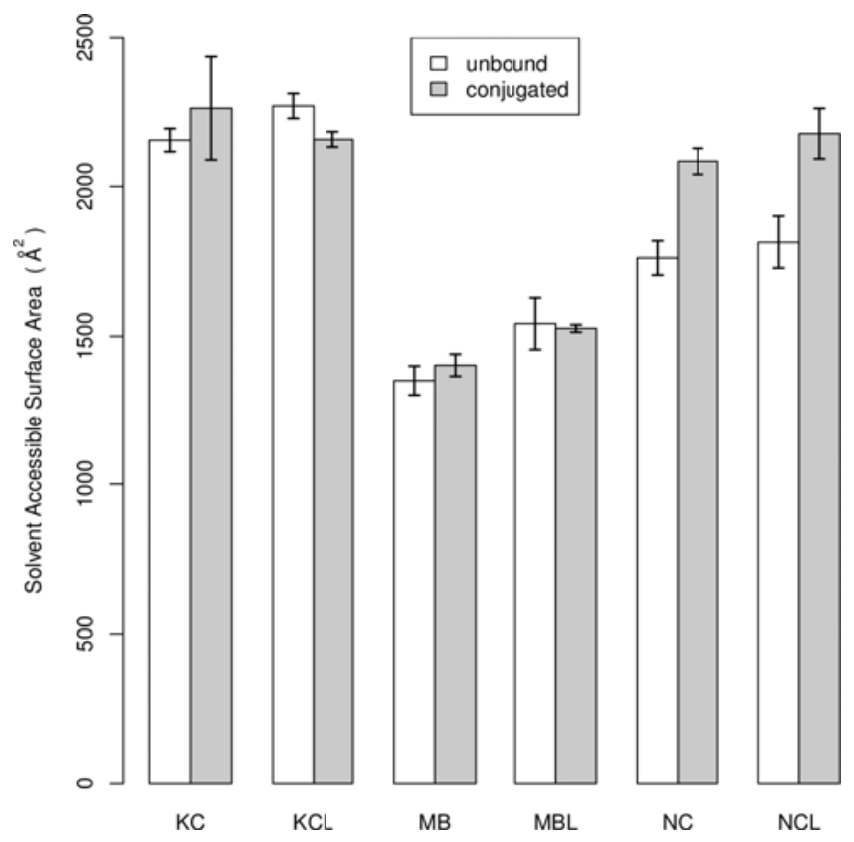

Figure 4 shows the conformational entropy of the $\mathrm{C} \alpha$ atoms for unbound and conjugated peptides. The conformational entropy is a measure of the overall peptide flexibility.

These results indicate that both gold NP conjugation and the peptide sequence affect peptide conformational flexibility. Five of the six peptides decrease flexibility when conjugated with a gold NP (this is expected since one end of the peptide is tethered to the NP surface) and that the amount of the decrease depends on the peptide sequence. There are two mechanisms for decreasing flexibility that are relevant for this study. The first (not calculated here) is due to the fact that one end of the peptide tethered to the NP surface and thus the peptide has reduced translational and rotational motion. The second (shown in Figures 4 and 5) is that the flexibility of the peptide chain itself decreases. Interestingly, the results suggest that the $\mathrm{KC}$ peptide increases flexibility when conjugated, in contrast to the other five peptides that decrease flexibility. This is because the $\mathrm{KC}$ peptide is more extended when conjugated as shown by the SASA results in Figure 3. Thus, for some peptide sequences, conjugation to a NP may increase the flexibility of the peptide. This could be important when designing peptides for nanoparticle conjugates for drug design since increasing the flexibility of the peptide may enhance the chances for interaction with the cellular media. 
Figure 4. Average conformational entropy for unbound (white) and conjugated (grey) cases for each peptide. The entropy is a measure of the overall peptide flexibility. Averages (bars) were computed for the $20 \mathrm{~ns}$ production simulation and uncertainties (error bars) were estimated as the standard deviation of block averages.

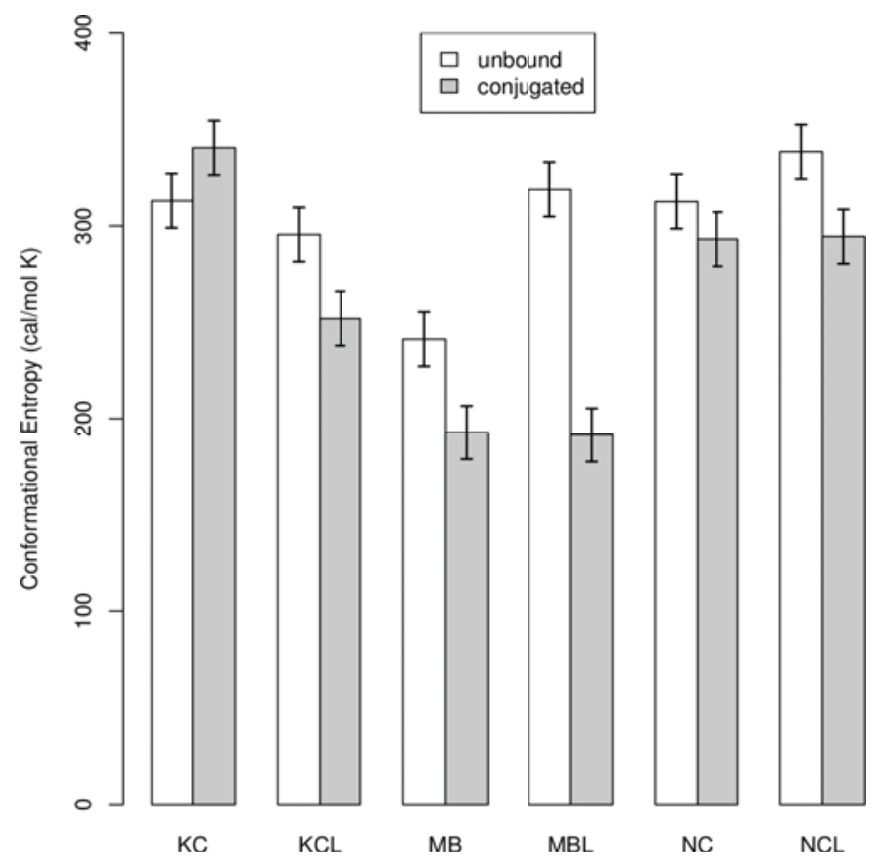

Figure 5 shows the RMSF of the $\mathrm{C} \alpha$ atoms for unbound and conjugated peptides. These results give insight into how the flexibility of each residue changes when conjugated to the gold NP. The MB and NC peptide results (Figure 5c,e) show that, even when the flexibility of the overall peptide decreases, the flexibility of some residues may still increase when conjugated.

Figure 5. Peptide average $\mathrm{C} \alpha$ root mean square fluctuation (RMSF) as a function of residue number for unbound (blue circles, solid) and conjugated (red diamonds, dashed) cases. Results were computed for the 20 ns production simulation.

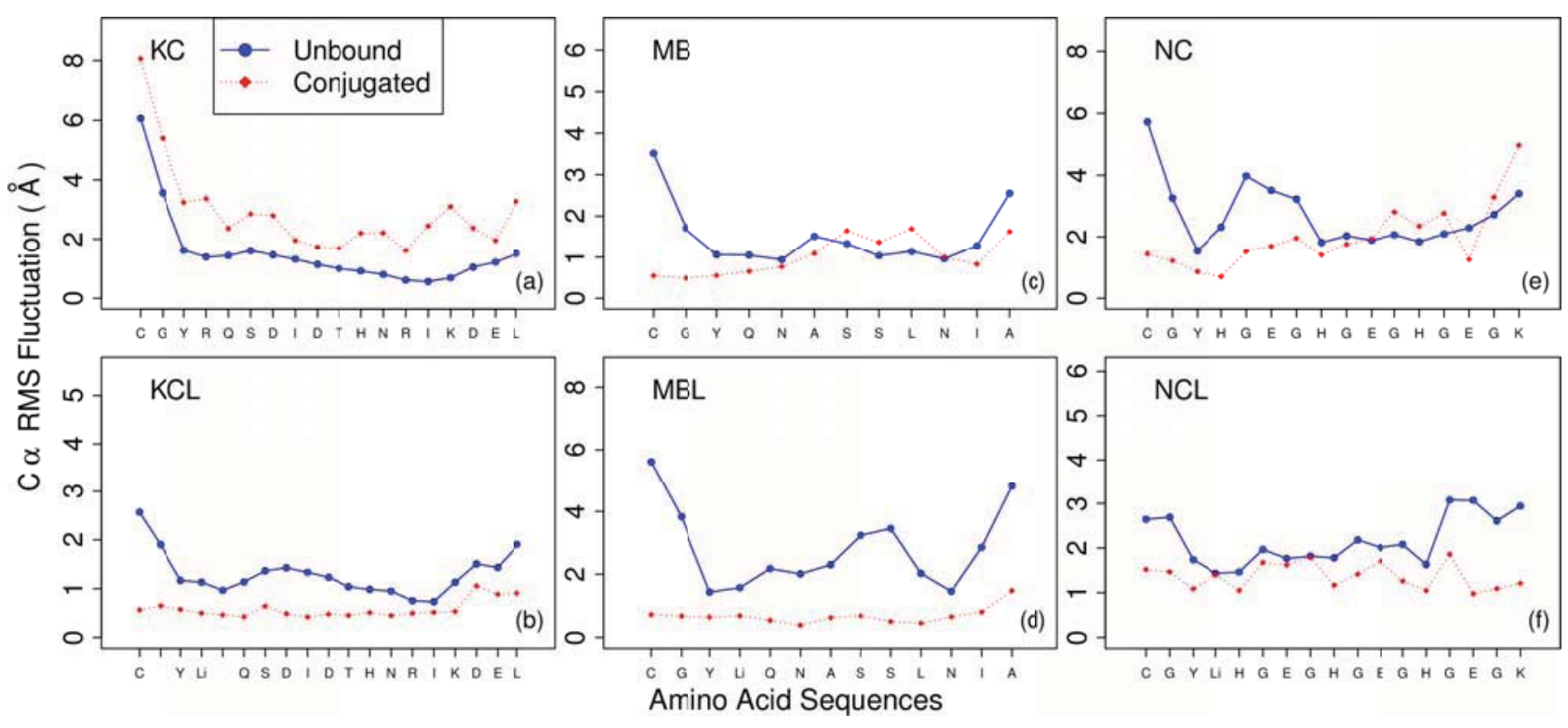


For the NC and NCL peptides there are several residues that are markedly less flexible when conjugated (Figure 5e,f). This is consistent with the reduced conformational entropy for NC and NCL when conjugated (Figure 4), however, this does not appear to be consistent with the SASA results (Figure 3) that show these peptides are more extended when conjugated. This apparent contradiction is resolved by observing the molecular motion during the production simulation where it is seen that the NC and NCL peptides adsorb to the surface of the gold NP at multiple points along the peptide (Figure 6).

The KC peptide results show that, in contrast to the other peptides studied here, conjugation to the gold NP increases the conformational entropy and RMSF of the peptide (Figures 4 and 5a). To test the robustness of this result we performed an independent $40 \mathrm{~ns}$ unbound simulation of the KC peptide starting with the structure from the last frame of the bound simulation. Results from this simulation showed that the most common structure, conformational entropy, RMSF, and SASA all returned to similar values when started from the bound structure compared to when starting with the PEP-FOLD structure (data not shown).

Figure 6. Snapshot of the NC peptide adsorbed to the surface of the gold NP. This adsorption also occurs for the NCL peptide (not shown). The sphere (yellow) is the sulfur atom that is bonded to the gold surface and is part of the cysteine residue (blue). We believe this adsorption occurs due to the lack of secondary structure in solution for these peptides.

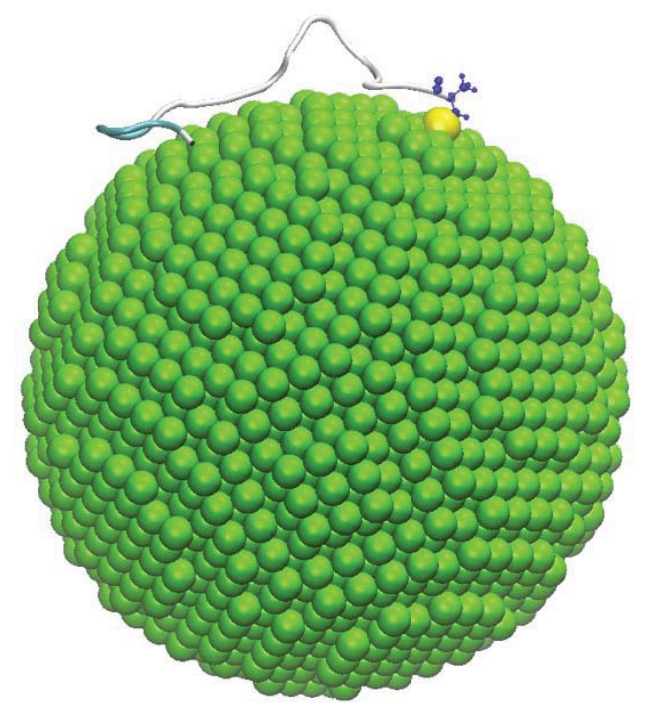

Our findings have important implications for anyone wishing to design nanoparticle conjugates for drug delivery. The NC and NCL peptides, which contain little to no secondary structure in solution, adsorb to the gold NP surface. This adsorption causes increased SASA (Figure 3) and decreased flexibility (Figures 4 and 5). None of the other four peptides, which do contain significant secondary structure in solution, adsorb to the NP surface. Also, the hydrophobicity of the NC and NCL peptides are lower than the other four. Thus, we believe that it is the lack of secondary structure that is allowing the NC and NCL peptides to adsorb. This result is important for designing nanoparticle conjugates for drug delivery because the conjugated peptides should not adsorb to the surface since this adsorption 
could reduce or eliminate specific desired interactions between the peptides and the cellular media. Thus, we suggest that when developing such nanoparticle conjugates the peptides should be designed to contain significant secondary structure in solution.

We note that since the current study uses a single peptide the results should be considered most relevant to NP conjugates with a low density of peptides. If the conjugate has a high density of peptides on the surface then peptide-peptide interactions could be important for determining peptide structure and dynamics.

\section{Conclusions}

We performed molecular dynamics simulation of six peptides to study the effect of gold NP conjugation on peptide structure and dynamics. For each peptide we tested two cases: a single unbound peptide in water, and a single peptide conjugated to a gold NP in water. Results show that, consistent with our hypothesis, the presence of gold NP does alter both the peptide structures and dynamics, and that the magnitude of the effect depends on the peptide sequence. Conjugated peptides typically have decreased conformational flexibility, and the amount of decrease depends on the amino acid sequence. However, it is possible for conjugation to increase the flexibility, as was the case with one of the peptides in this study. Conjugating the peptides to a gold NP can also result in more extended structures or more compact structures, depending on the amino acid sequence of the peptide.

Finally, we suggest that if one wishes to design peptides for nanoparticle conjugates for drug delivery the peptides should contain significant secondary structure in solution. This is because our results show that peptides with little to no secondary structure in solution tend to adsorb to the nanoparticle surface, potentially losing their ability specifically interact with cellular media.

\section{Acknowledgments}

We thank Rod A. Hill and his research group at University of Idaho for providing the peptide sequences and for motivating this study. This study was supported in part by grant P20RR01644807S1 from the National Institutes of Health. The content is solely the responsibility of the authors and does not necessarily represent the official views of the National Institutes of Health. Computational resources were provided in part by the Institute for Bioinformatics and Evolutionary Studies at University of Idaho.

\section{References}

1. Medintz, I.L.; Konnert, J.H.; Clapp, A.R.; Stanish, I.; Twigg, M.E.; Mattoussi, H.; Mauro, J.M.; Deschamps, J.R. A fluorescence resonance energy transfer-derived structure of a quantum dot-protein bioconjugate nanoassembly. Proc. Natl. Acad. Sci. U. S. A. 2004, 101, 9612-9617.

2. Liu, W.; Howarth, M.; Greytak, A.B.; Zheng, Y.; Nocera, D.G.; Ting, A.Y.; Bawendi, M.G. Compact biocompatible quantum dots functionalized for cellular imaging. J. Am. Chem. Soc. 2008, 130, 1274-1284.

3. Bruchez, M.J.; Moronne, M.; Gin, P.; Weiss, S.; Alivisatos, A.P. Semiconductor nanocrystals as fluorescent biological labels. Science 1998, 281, 2013-2016. 
4. Gao, X.; Yang, L.; Petros, J.A.; Marshall, F.F.; Simons, J.W.; Nie, S. In vivo molecular and cellular imaging with quantum dots. Curr. Opin. Biotechnol. 2005, 16, 63-72.

5. Neuberger, T.; Schöpf, B.; Hofmann, H.; Hofmann, M.; von Rechenberg, B. Superparamagnetic nanoparticles for biomedical applications: Possibilities and limitations of a new drug delivery system. J. Magn. Magn. Mater. 2005, 293, 483-496.

6. Daniel, M.-C.; Astruc, D. Gold nanoparticles: Assembly, supramolecular chemistry, quantumsize-related properties, and applications toward biology, catalysis, and nanotechnology. Chem. Rev. 2004, 104, 293-346.

7. Connor, E.; Mwamuka, J.; Gole, A.; Murphy, C.J.; Wyatt, M.D. Gold nanoparticles are taken up by human cells but do not cause acute cytotoxicity. Small 2005, 1, 325-327.

8. Male, K.B.; Lachance, B.; Hrapovic, S.; Sunahara, G.; Luong, J.H. Assessment of cytotoxicity of quantum dots and gold nanoparticles using cell-based impedance spectroscopy. Anal. Chem. 2008, 80, 5487-5493.

9. Allen, T.M. Ligand-targeted therapeutics in anticancer therapy. Nat. Rev. Cancer 2002, 2, 750-763.

10. Peer, D.; Karp, J.M.; Hong, S.; Farokhzad, O.C.; Margalit, R.; Langer, R. Nanocarriers as an emerging platform for cancer therapy. Nat. Nanotechnol. 2007, 2, 751-760.

11. Farokhzad, O.C.; Cheng, J.; Teply, B.A.; Sherifi, I.; Jon, S.; Kantoff, P.W.; Richie, J.P.; Langer, R. Targeted nanoparticle-aptamer bioconjugates for cancer chemotherapy in vivo. Proc. Natl. Acad. Sci. U. S. A. 2006, 103, 6315-6320.

12. Farokhzad, O.C.; Jon, S.; Khademhosseini, A.; Tran, T.-N.T.; LaVan, D.A.; Langer, R. Nanoparticle-aptamer bioconjugates: A new approach for targeting prostate cancer cells. Cancer Res. 2004, 64, 7668-7672.

13. Brown, S.D.; Nativo, P.; Smith, J.-A.; Stirling, D.; Edwards, P.R.; Venugopal, B.; Flint, D.J.; Plumb, J.A.; Graham, D.; Wheate, N.J. Gold nanoparticles for the improved anticancer drug delivery of the active component of oxaliplatin. J. Am. Chem. Soc. 2010, 132, 4678-4684.

14. Cheng, Y.; Samia, A.C.; Meyers, J.D.; Panagopoulos, I.; Fei, B.; Burda, C. Highly efficient drug delivery with gold nanoparticle vectors for in vivo photodynamic therapy of cancer. J. Am. Chem. Soc. 2008, 130, 10643-10647.

15. Thomas, M.; Klibanov, A.M. Conjugation to gold nanoparticles enhances polyethylenimine's transfer of plasmid DNA into mammalian cells. Proc. Natl. Acad. Sci. U. S. A. 2003, 100, 9138-9143.

16. Joshi, H.M.; Bhumkar, D.R.; Joshi, K.; Pokharkar, V.; Sastry, M. Gold nanoparticles as carriers for efficient transmucosal insulin delivery. Langmuir 2006, 22, 300-305.

17. Aubin-Tam, M.-E.; Hamad-Schifferli, K. Gold nanoparticle-cytochrome c complexes: The effect of nanoparticle ligand charge on protein structure. Langmuir 2005, 21, 12080-12084.

18. Aubin-Tam, M.-E.; Hamad-Schifferli, K. Structure and function of nanoparticle-protein conjugates. Biomed. Mater. 2008, 3, 034001.

19. Verma, A.; Nakade, H.; Simard, J.M.; Rotello, V.M. Recognition and stabilization of peptide $\alpha$-helices using templatable nanoparticle receptors. J. Am. Chem. Soc. 2004, 126, 10806-10807.

20. Verma, A.; Rotello, V.M. Surface recognition of biomacromolecules using nanoparticle receptors. Chem. Commun. 2005, 303-312. 
21. Slocik, J.M.; Naik, R.R. Probing peptide-nanomaterial interactions. Chem. Soc. Rev. 2010, 39, 3454-3463.

22. So, C.R.; Kulp, J.L., III; Oren, E.E.; Zareie, H.; Tamerler, C.; Evans, J.S.; Sarikaya, M. Molecular recognition and supramolecular self-assembly of a genetically engineered gold binding peptide on Au $\{111\}$. ACS Nano 2009, 3, 1525-1531.

23. Braun, R.; Sarikaya, M.; Schulten, K. Genetically engineered gold-binding polypeptides: Structure prediction and molecular dynamics. J. Biomater. Sci. Polym. Ed. 2002, 13, 747-757.

24. Hoefling, M.; Iori, F.; Corni, S.; Gottschalk, K.-E. Interaction of amino acids with the Au (111) surface: Adsorption free energies from Molecular dynamics simulations. Langmuir 2010, 26, 8347-8351.

25. Vila Verde, A.; Acres, J.M.; Maranas, J.K. Investigating the specificity of peptide adsorption on gold using molecular dynamics simulations. Biomacromolecules 2009, 10, 2118-2128.

26. Lee, O.-S.; Schatz, G.C. Interaction between DNAs on a gold surface. J. Phys. Chem. C 2009, $113,15941-15947$.

27. Lee, O.-S.; Schatz, G.C. Molecular dynamics simulation of DNA-functionalized gold nanoparticles. J. Phys. Chem. C 2009, 113, 2316-2321.

28. Largo, J.; Starr, F.W.; Sciortino, F. Self-assembling DNA dendrimers: A numerical study. Langmuir 2007, 23, 5896-5905.

29. Wang, G.; Papasani, M.R.; Hrdlicka, P.J.; Hill, R.A. Role of serum proteins in the cellular uptake of gold-peptide nanoconjugates. NSTI-Nanotech 2010, 3, 304-307.

30. Hill, R. Department of Animal and Veterinary Science, University of Idaho, Moscow, ID 83844, USA. Personal Communication, 2010.

31. Maupetit, J.; Derreumaux, P.; Tuffery, P. PEP-FOLD: An online resource for de novo peptide structure prediction. Nucleic Acids Res. 2009, 37, W498-W503.

32. Jiang, J.; Lu, W.; Luo, Y. Length dependence of coherent electron transportation in metalalkanedithiol-metal and metal-alkanemonothiol-metal junctions. Chem. Phys. Lett. 2004, 400, $336-340$.

33. Phillips, J.C.; Braun, R.; Wang, W.; Gumbart, J.; Tajkhorshid, E.; Villa, E.; Chipot, C.; Skeel, R.D.; Kalé, L.; Schulten, K. Scalable molecular dynamics with NAMD. J. Comput. Chem. 2005, 26, 1781-1802.

34. Jorgensen, W.L.; Chandrasekhar, J.; Madura, J.D.; Impey, R.W.; Klein, M.L. Comparison of simple potential functions for simulating liquid water. J. Chem. Phys. 1983, 79, 926-935.

35. Brooks, B.R.; Bruccoleri, R.E.; Olafson, B.D.; States, D.J.; Swaminathan, S.; Karplus, M. CHARMM: A program for macromolecular energy, minimization, and dynamics calculations. J. Comput. Chem. 1983, 4, 187-217.

36. Miao, L.; Seminario, J.M. Molecular dynamics simulations of the vibrational signature transfer from a glycine peptide chain to nanosized gold clusters. J. Phys. Chem. C 2007, 111, 8366-8371.

37. Iori, F.; Di Felice, R.; Molinari, E.; Corni, S. GolP: An atomistic force-field to describe the interaction of proteins with $\mathrm{Au}(111)$ surfaces in water. J. Comput. Chem. 2009, 30, 1465-1476.

38. Heinz, H.; Vaia, R.; Farmer, B.L.; Naik, R.R. Accurate simulation of surfaces and interfaces of face-centered cubic metals using $12-6$ and 9-6 lennard-jones potentials. J. Phys. Chem. C 2008, $112,17281-17290$. 
39. van Gunsteren, W.F.; Berendsen, H.J.C.; Rullmann, J.A.C. Stochastic dynamics for molecules with constraints Brownian dynamics of normal-alkanes. Mol. Phys. 1981, 44, 69-95.

40. Martyna, G.J.; Tobias, D.J.; Klein, M.L. Constant pressure molecular dynamics algorithms. J. Chem. Phys. 1994, 101, 4177-4189.

41. Feller, S.E.; Zhang, Y.; Pastor, R.W.; Brooks, B.R. Constant pressure molecular dynamics simulation: The Langevin piston method. J. Chem. Phys. 1995, 103, 4613.

42. Ryckaert, J.-P.; Ciccotti, G.; Berendsen, H.J.C. Numerical integration of the cartesian equations of motion of a system with constraints: Molecular dynamics of n-alkanes. J. Comput. Phys. 1977, 23, $327-341$.

43. Darden, T.; York, D.; Pedersen, L. Particle mesh Ewald: An N.log(N) method for Ewald sums in large systems. J. Chem. Phys. 1993, 98, 10089-10092.

44. Humphrey, W.; Dalke, A.; Schulten, K. VMD: Visual molecular dynamics. J. Mol. Graphics 1996, 14, 33-38.

45. R Development Core Team. $R: A$ Language and Environment for Statistical Computing; R Foundation for Statistical Computing: Vienna, Austria, 2008.

46. van der Spoel, D.; Lindahl, E.; Hess, B.; Groenhof, G.; Mark, A.E.; Berendsen, H.J.C. GROMACS: Fast, flexible, and free. J. Comput. Chem. 2005, 26, 1701-1718.

47. Andricioaei, I.; Karplus, M. On the calculation of entropy from covariance matrices of the atomic fluctuations. J. Chem. Phys. 2001, 115, 6289-6292.

48. Schlitter, J. Estimation of absolute and relative entropies of macromolecules using the covariance matrix. Chem. Phys. Lett. 1993, 215, 617-621.

49. Schäfer, H.; Mark, A.E.; van Gunsteren, W.F. Absolute entropies from molecular dynamics simulation trajectories. J. Chem. Phys. 2000, 113, 7809-7817.

50. Bennett, W.S., Jr.; Steitz, T.A. Glucose-induced conformational change in yeast hexokinase. Proc. Natl. Acad. Sci. U. S. A. 1978, 75, 4848-4852.

(C) 2012 by the authors; licensee MDPI, Basel, Switzerland. This article is an open access article distributed under the terms and conditions of the Creative Commons Attribution license (http://creativecommons.org/licenses/by/3.0/). 